\title{
Ploidy and DNA index as prognostic factors in resected pancreatic ductal adenocarcinoma - a review of the literature
}

\author{
Marek Durlik ${ }^{1,2}$, Julia Tuchalska-Czurońn ${ }^{1,3}$ \\ ${ }^{1}$ Department of Surgical Research and Transplantology, Mossakowski Medical Research Centre, Polish Academy of Sciences, \\ Warsaw, Poland \\ ${ }^{2}$ Clinical Department of Gastroenterological Surgery and Transplantation, Central Clinical Hospital of the Ministry of Interior, \\ Warsaw, Poland \\ ${ }^{3}$ Diagnostic Radiology Department, Central Clinical Hospital of the Ministry of Interior, Warsaw, Poland
}

Prz Gastroenterol 2014; 9 (6): 313-316 DOI: $10.5114 /$ pg.2014.47892

Key words: pancreatic cancer, ploidy, DNA index.

Address for correspondence: Marek Durlik MD, PhD, Assoc. Prof., Clinical Department of Gastroenterological Surgery and Transplantation, Central Clinical Hospital of the Ministry of Interior, 137 Wołoska St, 02-507 Warsaw, Poland, phone: +48 2250810 20, e-mail: dyrekcja@cskmswia.pl, mdurlik@post.home.pl

\begin{abstract}
In Poland, pancreatic cancer is the seventh most common cause of cancer-related death amongst men and the sixth amongst women. Pancreatic cancer has an extremely poor prognosis. Radical surgery still remains the only way of curing pancreatic cancer, and this is possible to perform in just $20 \%$ of cases, i.e. those that present localised disease upon diagnosis. An average survival of 20 months post-resection and adjuvant chemotherapy has been observed in 10-15\% of patients with tumours limited to the pancreas at the time of presentation. It is necessary to define strong prognostic factors to determine individual treatment and prognosis. In this paper we submit a review of the literature concerning the prognostic impact of the ploidy and the DNA index on the survival of patients who underwent resection of pancreatic ductal adenocarcinoma. However, the presented studies have produced conflicting results. Pancreatic cancer still remains a great challenge in medicine.
\end{abstract}

\section{Introduction}

According to the National Cancer Registry, in Poland there are over 3000 new cases of pancreatic cancer per year. Pancreatic cancer (PC) is the seventh most common cause of cancer-related death amongst men and the sixth amongst women.

In total $90 \%$ of pancreatic cancers are ductal adenocarcinomas (PDAC), which are known for their extremely poor prognosis. In most cases, at the time of diagnosis this malignant neoplasm is unresectable because of distant metastases. Seventy-four percent of patients die within the first year of diagnosis. Only about $20 \%$ of pancreatic cancer patients qualify for surgery, which is the only widely accepted curative treatment method. An average survival of $\sim 20$ months post-resection and adjuvant chemotherapy has been observed for $10-15 \%$ of patients with tumours limited to the pancreas at the time of presentation [1]. Gemcitabine is the standard chemo- therapeutic agent in pancreatic cancer. Unfortunately, two-thirds of pancreatic tumours display low expression of human equilibrative nucleoside transporter 1 (hENT1), which mediates cellular entry of the drug, and do not respond to gemcitabine therapy. Other mechanisms of resistance to gemcitabine are deficient deoxycytidine kinase activity (these kinase phosphorylates gemcitabine to active metabolites) and overexpression of ribonucleotide reductase (one of the active metabolites of gemcitabine works by blocking these enzymes) [2].

\section{Prognostic factors in pancreatic cancer}

Many studies into the prognostic factors in pancreatic cancer have been conducted for a number of years. Prognostic factors allow clinicians to select the individual treatment strategy, and they can be divided into several groups, including the following: widely available blood tests, tumour markers, immunohistochemical 
markers, histological staging and grading, the influence of administered therapy, clinical condition of the patient, and demographic factors. It is a broad subject, so we decided to highlight the most interesting data. Chiang et al. reported that patients with a better nutritional status (measured as having an albumin level greater than $3.5 \mathrm{~g} / \mathrm{dl}$ ), radical resection, early tumour stage, and better-differentiated tumours were associated with favourable survival [3]. Of great interest is a study carried out in 2012 by Sanjay et al. which showed that a pre-operative elevated serum C-reactive protein (CRP) level and raised post-operative lymph node ratio (ratio of positive over excised lymph nodes) represent significant independent prognostic factors that predict poor prognosis in patients undergoing curative resection for pancreatic ductal adenocarcinoma [4]. The results of the study conducted in 2011 by de Jong et al. are quite remarkable. The authors analysed 1697 cases of patients who underwent curative pancreaticoduodenectomy because of pancreatic adenocarcinoma in terms of tumour size, and they concluded that the cut-off value of $2 \mathrm{~cm}$ is not independently associated with the outcome; however, tumour size is strongly associated with the risk of other adverse prognostic factors. The effect of size on prognosis is largely attributable to these other biological factors rather than the size of the tumour itself [5]. Smith et al. performed a meta-analysis of immunohistochemical prognostic markers in resected pancreatic cancer. In this meta-analysis vascular endothelial growth factor (VEGF) emerged as the most potentially informative prognostic marker; $\mathrm{Bcl}-2$, bax and p16 also returned significant overall survival differences, but in smaller patient series due to a lack of evaluable literature [6]. Boyd et al. reported that depression was a significant predictor of survival in patients with pancreatic cancer who underwent surgical resection (hazard ratio, 1.34; 95\% confidence interval, 1.04-1.73) [7]. Wang et al. showed that patients with metabolic syndrome and pancreatic cancer had later disease staging and a poorer histological grade. They also demonstrated significantly poorer survival [8].

\section{Genetic background of pancreatic cancer}

A number of studies on the molecular level have been conducted to gain deeper insight into cancer biology. Analyses have shown that the most common genetic alterations identified to date in pancreatic cancer are activation of the KRAS oncogene and inactivation of the CDKN2/p16, TP53, and DPC4 tumour suppressor genes. Mutation of KRAS occurs in $80-95 \%$ of cases and mutation of CDKN2 in 75-95\%. The association of these two mutations is not frequent in other cancers, which is why it is called the "molecular fingerprint" of pancreatic cancer. Mutation of the TP53 suppressor gene affects more than half of PC tumours. Another suppressor gene, DPC4 (deleted in pancreatic cancer 4), is mutated in $50-55 \%$ of cases $[9,10]$. Oshima et al. retrospectively analysed the relationship between selected clinical parameters and immunohistochemically detected expression of CDKN2A/p16, TP53, and DPC4. Abnormal immunolabelling of p53 was significantly associated with tumour dedifferentiation $(p=0.022)$ and the presence of locoregional recurrence $(p=0.020)$. Loss of CDKN2/p16 immunolabelling was associated with lymphatic invasion ( $p=0.012$ ) and postoperative widespread metastases $(p<0.001)$. A significant correlation was found between DPC4 immunolabelling and tumour size $(p=0.006)$, lymphatic invasion $(p=0.033)$, and lymph node metastasis $(p=0.006)$. Smith et al. conducted a meta-analysis related to resected PC, which confirmed a prognostic value for CDKN2/ p16, but neither TP53 nor DPC4/smad4 was found to represent significant prognostic factors [6]. In studies of the molecular background of PC, authors have also described mutations of SWI/SNF (35\% of cases), APC, MCC, DCC, BRCA2, and RB1 [9-12].

\section{Ploidy and DNA index as prognostic factors in pancreatic cancer}

Ploidy is the number of sets of chromosomes in the nucleus of a biological cell. A typical human somatic cell contains 46 chromosomes $(2 n=46)$. This applies to somatic cells in the G0/G1 phase of mitotic cell cycle. Cells are described according to the number of sets present: haploid (1 set), diploid (2 sets), triploid (3 sets), tetraploid (4 sets), pentaploid (5 sets), hexaploid (6 sets), heptaploid/septaploid (7 sets), etc. The generic term polyploid is frequently used to describe cells with three or more sets of chromosomes (triploid or higher ploidy). Euploidy is the state of a cell or organism having an integral multiple of the monoploid number. For example, a human cell has 46 chromosomes, which is an integer multiple of the monoploid number, 23. A human with abnormal, but integral, multiples of this full set (e.g. 69 chromosomes) would also be considered as euploid. Aneuploidy is the state of not having euploidy. The DNA index is the mean nuclear DNA content of the G0/G1 compartment of the neoplastic cells population divided by the DNA content of the GO/G1 compartment of the known diploid reference cells.

Of interest to this paper is the relationship between the survival of patients operated upon because of pancreatic ductal adenocarcinoma and cancer cell ploidy (DNA index). Here, we can ask if there is any relationship between the resectability of pancreatic cancer and 
Table I. Studies on the relationship between ploidy/DNA index and survival of patients operated upon because of pancreatic ductal adenocarcinoma

\begin{tabular}{|c|c|c|c|c|c|c|c|c|c|}
\hline Authors & $\begin{array}{l}\text { Design } \\
\text { of study }\end{array}$ & $\begin{array}{c}\text { No. of } \\
\text { patients }\end{array}$ & $\begin{array}{l}\text { Diploid } \\
(\%)\end{array}$ & $\begin{array}{c}\text { Non-diploid } \\
(\%)\end{array}$ & $\begin{array}{l}\text { Survival in } \\
\text { diploid group } \\
\text { [months] }\end{array}$ & $\begin{array}{l}\text { Survival in non } \\
\text { diploid group } \\
\text { [months] }\end{array}$ & $\begin{array}{l}\text { Value } \\
\text { of } p\end{array}$ & $\begin{array}{l}\text { DNA } \\
\text { index }\end{array}$ & $\begin{array}{l}\text { Value } \\
\text { of } p\end{array}$ \\
\hline Kamphues et al. & Prospect. & 61 & 24.6 & 75.6 & 29.2 & 13.5 & 0.252 & 1.9 & 0.026 \\
\hline Yeo et al. & Retrosp. & 119 & 43 & 57 & 24 & 11.5 & 0.0002 & - & - \\
\hline Berczi et al. & Retrosp. & 22 & 63.6 & 36.4 & 10 & 8 & $>0.05$ & - & - \\
\hline Bui et al. & Retrosp. & 20 & 35 & 65 & 17 & 7.5 & $<0.03$ & - & - \\
\hline Hyoty et al. & Retrosp. & 53 & 79 & 21 & 14 & 9 & 0.11 & $1.0-3.21$ & - \\
\hline Allison et al. & Retrosp. & 47 & 40 & 60 & 25 & 10.5 & 0.003 & - & - \\
\hline
\end{tabular}

DNA content. In the prospective study He et al. showed that there is a high correlation between the resectability of pancreatic cancers and their DNA ploidy status. In this study, a total of 36 patients with pancreatic adenocarcinoma were divided into resectable and unresectable groups. Statistical significance was found in DNA ploidy in both groups $(p<0.05)$. The rates of diploid/ tetraploid and aneuploid were $66.7 \%$ and $33.3 \%$ in the resectable group, respectively, and $38.9 \%$ and $62.1 \%$ in the unresectable group, respectively $(p<0.05)$ [13].

The following is a presentation of studies on the relationship between the survival of patients with resectable pancreatic ductal adenocarcinoma and their ploidy/DNA index. The results of similar studies are summarised in Table I.

Kamphues et al. reported that the DNA index represents an independent predictive marker in patients with pancreatic head cancer. In this prospective study, the DNA ploidy and the DNA index of 61 patients operated upon because of pancreatic head cancer were evaluated by DNA image cytometry. Of these, 15 tumours (24.6\%) were identified as diploid and 46 (75.6\%) as nondiploid. The median DNA index in the entire cohort was 1.9 (range: 1.0-2.5). The average survival of the study cohort was 17.5 months. In multivariate survival analysis, DNA index $(p=0.026)$ and lymph node status $(p=0.007)$ were identified as independent prognostic factors predicting the survival of the study cohort [14].

Yeo et al. showed that DNA content is one of the strongest predictors of outcome after pancreaticoduodenectomy in patients with adenocarcinoma of the head of the pancreas. Of the 119 patients whose tumours were analysed for DNA content, 51 (43\%) had diploid tumours and 68 (57\%) had aneuploid tumours. Patients with diploid tumours had a median survival of 24 months and a 5-year survival of 39\%, significantly better than the average survival of 11.5 months and 5 -year survival of $8 \%$ observed in patients with aneuploid tumours ( $p=0.0002)$ [15].
Hyöty et al. reported that there is no correlation between ploidy/DNA index and survival in patients who had undergone resection of pancreatic cancer. The authors analysed 53 cases of resected pancreatic ductal adenocarcinoma. The DNA index ranged from 1.00 to 3.21. Aneuploidy was detected in 11 cases (21\%). After excluding 8 patients who did not die from cancer, the average survival was 13 months. The survival of the patients with DNA-aneuploid tumours (median 9 months) did not differ significantly from that of the patients with diploid tumours (median 14 months), $p=0.11$ [16].

In contrast to this study, Allison et al. obtained totally different results. In their study the DNA content of 47 adenocarcinomas arising in the head of the pancreas from patients who had undergone successful pancreatoduodenectomy was measured. Of the 47 tumours, 19 (40\%) were diploid and 28 (60\%) were aneuploid cancers. The 19 patients with diploid cancers had an average survival time of 25 months. Average survival of the 28 patients with aneuploid cancers was 10.5 months. This difference was statistically significant $(p=0.003)$. A multivariate life table regression analysis demonstrated that the ploidy is an independent prognostic factor for patients with adenocarcinoma of the head of the pancreas, who have successfully undergone a pancreaticoduodenectomy [17].

The study conducted by Herrera et al. was slightly different. The authors hypothesised that if the ploidy pattern correlated to survival, recognition of this pattern should be more apparent in long-term survivors in a population of patients undergoing resection for pancreatic ductal adenocarcinoma. In this retrospective study, 72 patients were divided into two groups according to the length of survival: the long-term survivors - group I (19 patients who survived 3 or more years after operation) and the short-term survivors - group II (43 patients who died within 12 months after resection). All hospital deaths (within 30 days of operation) 
and patients who survived for at least 1 but less than 3 years after operation were excluded from this study. Because of poor-quality histograms the authors were unable to interpret 10 of the 72 samples evaluated. These patients were excluded from analysis. In 30 specimens (48\%), the nuclear DNA pattern was diploid, whereas 32 were nondiploid (2 tetraploid and 30 aneuploid). In group I, 12 patients (63\%) had DNA diploid tumours and 7 (37\%) had a nondiploid DNA pattern, compared with 18 (42\%) and 25 (58\%), respectively, in group II. The DNA index of the 19 patients with long-term survival ranged from 1 to 1.86 (mean 1.1) and that of the 43 patients with short-term survival ranged from 1 to 2 (mean 1.2). There were no significant differences in the number of diploid/nondiploid patterns or DNA indices between the two groups [18].

\section{Conclusions}

Despite significant advances in the surgical and chemotherapeutic management of patients with pancreatic cancer in recent years, the prognosis is extremely poor. That is why many studies have focused on the reasons for the aggressive biology of pancreatic cancer and the prognostic values of different factors. Studies evaluating the prognostic impact of the ploidy and the DNA index have produced conflicting results. However, most of these studies were retrospective and based on the analysis of paraffin-embedded archival material, and therefore provided only limited evidence. Further, large, prospective studies are needed, and pancreatic cancer still remains a great challenge in medicine.

\section{References}

1. Kaur S, Baine MJ, Jain M, et al. Early diagnosis of pancreatic cancer: challenges and new developments. Biomark Med 2012; 6: 597-612.

2. Ansari D, Tingstedt B, Andersson R. Pancreatic cancer - cost for overtreatment with gemcitabine. Acta Oncol 2013; 52: 1146-51.

3. Chiang KC, Yeh CN, Ueng SH, et al. Clinicodemographic aspect of resectable pancreatic cancer and prognostic factors for resectable cancer. World J Surg Oncol 2012; 10: 77.

4. Sanjay $\mathrm{P}$, de Figueiredo RS, Leaver $\mathrm{H}$, et al. Preoperative serum C-reactive protein levels and post-operative lymph node ratio are important predictors of survival after pancreaticoduodenectomy for pancreatic ductal adenocarcinoma. JOP 2012; 13: 199-204.

5. de Jong MC, Li F, Cameron JL, et al. Re-evaluating the impact of tumour size on survival following pancreaticoduodenectomy for pancreatic adenocarcinoma. J Surg Oncol 2011; 103: 656-62.

6. Smith RA, Tang J, Tudur-Smith C, et al. Meta-analysis of immunohistochemical prognostic markers in resected pancreatic cancer. Br J Cancer 2011; 104: 1440-51.
7. Boyd CA, Benarroch-Gampel J, Sheffield KM, et al. The effect of depression on stage at diagnosis, treatment, and survival in pancreatic adenocarcinoma. Surgery 2012; 152: 403-13.

8. Wang DS, Wang ZQ, Zhang L, et al. Are risk factors associated with outcomes in pancreatic cancer? PLoS One 2012; 7 : e41984.

9. Oshima M, Okano K, Muraki S, et al. Immunohistochemically detected expression of 3 major genes (CDKN2A/p16, TP53, and SMAD4/DPC4) strongly predicts survival in patients with resectable pancreatic cancer. Ann Surg 2013; 258: 336-46.

10. Howe JR, Conlon KC. The molecular genetics of pancreatic cancer. Surg Oncol 1997; 6: 1-18.

11. Shain AH, Giacomini CP, Matsukuma K, et al. Convergent structural alterations define SWItch/Sucrose NonFermentable (SWI/ SNF) chromatin remodeler as a central tumour suppressive complex in pancreatic cancer. Proc Natl Acad Sci U S A 2012; 109: E252-9.

12. Delpu Y, Hanoun N, Lulka H, et al. Genetic and epigenetic alterations in pancreatic carcinogenesis. Curr Genomics 2011; 12: $15-24$.

13. He YC, Peng W, Qiao JG, et al. Relationship between nuclear morphometry, DNA content and resectability of pancreatic cancer. World J Gastroenterol 2003; 9: 1863-5.

14. Kamphues C, Al-Abadi H, Dürr A, et al. DNA index as a strong prognostic factor in patients with adenocarcinoma of the pancreatic head: results of a 5-year prospective study. Pancreas 2013; 42: 807-12.

15. Yeo CJ, Cameron JL, Lillemoe KD, et al. Pancreaticoduodenectomy for cancer of the head of the pancreas. 201 patients. Ann Surg 1995; 221: 721-33.

16. Hyöty M, Visakorpi T, Kallioniemi OP, et al. Prognostic value of analysis of DNA in pancreatic adenocarcinoma by flow cytometry. Eur J Surg 1991; 157: 595-600.

17. Allison DC, Bose KK, Hruban RH, et al. Pancreatic cancer cell DNA content correlates with long-term survival after pancreatoduodenectomy. Ann Surg 1991; 214: 648-56.

18. Herrera MF, van Heerden JA, Katzmann JA, et al. Evaluation of DNA nuclear pattern as a prognostic determinant in resected pancreatic ductal adenocarcinoma. Ann Surg 1992; 215: 120-4.

Received: 11.11.2013

Accepted: 30.11 .2013 